\title{
Energy Conservation in Mobile Ad Hoc Network Using Energy Efficient Scheme and Magnetic Resonance
}

\author{
Manish Bhardwaj*, Ashish Bansal \\ Computer Science and Engineering Department, SRM University, Ghaziabad, India
}

Email address:

Aapkaapna13@gmail.com (M. Bhardwaj), ashishbansalmohan@gmail.com (A. Bansal)

To cite this article:

Manish Bhardwaj, Ashish Bansal. Energy Conservation in Mobile Ad Hoc Network Using Energy Efficient Scheme and Magnetic

Resonance. Advances in Networks. Special Issue: Secure Networks and Communications. Vol. 3, No. 3-1, 2015, pp. 34-39.

doi: $10.11648 /$ j.net.s.2015030301.15

\begin{abstract}
A mobile ad hoc network is a collection of wireless mobile nodes that communicate with one another without any fixed networking infrastructure. Since the nodes in this network are mobile, the energy management and energy conservation become very critical in mobile ad hoc network. The nodes in this network have limited battery power and limited computational power with a small amount of memory. Such nodes must conserve energy during routing to prolong their usefulness and increase network lifetime. This research paper proposes a scheme that takes into consideration the energy awareness during route selection. This scheme observes power status of each and every node in the topology and further ensures the fast selection of routes with minimal efforts and faster recovery. Along with this scheme also introduce the concept of wirelessly recharge the nodes of the networks. The scheme is incorporated with the AODV protocol and introduces new protocol EEAODV the performance has been studied through simulation over NS-2.34.
\end{abstract}

Keywords: Ad Hoc Networks, AODV, EEAODV, Topology

\section{Introduction}

A mobile ad hoc network is an autonomous system of mobile hosts which are free to move around randomly and organize themselves arbitrarily. This is viewed as suitable systems which can support some specific applications as virtual classrooms, military communications, emergency search and rescue operations, data acquisition in hostile environments, communications set up in exhibitions, conferences and meetings, in battle field among soldiers to coordinate defense or attack, at airport terminals for workers to share files etc. In ad hoc networks, nodes can change position quite frequently. The nodes in an ad hoc network can be laptops, PDA or palm tops etc. These are often limited in resources such as CPU capacity, storage capacity, battery power and bandwidth. Each node participating in the network acts both as a router and as a host and must therefore be willing to transfer packets to other nodes. For this purpose, a routing protocol should try to minimize control traffic [1]. There is limitation of battery life and in an ad hoc environment, battery is most commonly used.

The concept of energy as one of the deciding factor in route selection can be crucial in root discovery and root repair phase. Rest of the paper is organized as follows. Section 2 illustrates the energy related issues of routing protocols in mobile ad hoc network. New protocol description and system methodology is presented in section 3 . Brief the concept of wirelessly recharging of nodes of a network, Performance evaluation of new protocol EEAODV using network simulator NS-2.34 is presented in section 4 and conclusion with future scope is given in section 5 .

\section{Issues and Related Work}

The lack of a centralized authority complicates the problem of Medium Access Control (MAC) in mobile ad hoc networks. The medium access regulation procedures have to be enforced in a distributed and hence collaborative fashion by mobile nodes. In the shared broadcast medium transmission of packets from distinct mobile nodes are prone to collision. This contention based medium access results in retransmissions and appreciable delays. The performance of 
the MAC scheme affects the routing protocol adversely and consequently the energy consumption for packet transmission and reception increases [2].

On-demand routing is composed of route discovery and route maintenance (Royer E. M. and Toh C. K., 1999). In route discovery, a source uses flooding to find a route to its destination. The large number of packets generated by flooding consumes energy of nodes unnecessarily. The transit nodes, upon receiving a query, learn the path to the source and enter the route in their forwarding tables. The destination node responds using the path traversed by the query. Route maintenance is responsible for reacting to topological changes in the network, and its implementation differs from one algorithm to the other. On-demand protocols include schemes like Ad hoc On-demand Distance Vector routing (AODV) (Perkins and Royer, 1999) and Dynamic Source Routing (DSR) (Johnson and Maltz, 1996). In these protocols, route discovery and maintenance may become inefficient under heavy network load since intermediate nodes will have a higher probability of moving due to the delay in packet transmissions attributed to MAC contention. Routes have a higher probability of breaking as a result of mobility. The rediscovery or repair of routes wastes battery power. The flooding of route request and route reply packets in on-demand routing protocols may result in considerable energy drain. Every station that hears the route request broadcasts will consume an amount of energy proportional to the size of the broadcast packet. In addition, stations that hear a corrupted version of a broadcast packet will still consume some amount of energy. In a multi-hop ad hoc network, nodes must always be ready and willing to receive traffic from their neighbors. All the nodes unnecessarily consume power due to reception of the transmissions of their neighbors. This wastes an extensive amount of the total consumed energy throughout the lifetime of a node.

Recent developments related to energy on AODV protocol have been taken into consideration. AODV is an improvement on DSDV which aims at reducing system wide broadcasts. In this protocol routes are discovered ondemand basis and maintain these routes only when these are in use. There exist two energy efficient algorithms based on AODV as: AODVE and AODVM. In AODVE, to increase the lifetime routing is based on the minimum remaining energy metric and that route is selected in which there is a maximum of minimum remaining energy (MIN_RE) and this field is added in RREQ as well as in the RREP. Other parameters are same as in AODV. Similar to AODVE, the latter AODVM considers the residual energy but it also considers the hop count value. It increases the lifetime of a network by arranging almost all nodes to involve in data transfer. It also shows improvement in delay and energy consumption of node. In EEAODV protocol, the energy state of each node as well as of the entire network has been considered. New field is added to the RREQ message which carries the collected remaining energy of nodes participating between source and the destination. In this, destination node does not give an immediate reply to the request but waits for some time and in the mean time, calculate the mean energy of the network and this is stored in each node. In case of a new route, this mean energy is then compared with the energy remaining in the node and if it is less, then RREQ message is delayed by some time and by this the entire lifetime is extended. In energy aware AODV protocol, routing is done in the similar way as in ADOV but link breakage is detected by Signal-toInterference Ratio (SIR). Cross layer interaction is also used by which physical layer can give information about link state to the network layer. Directional antennas are also used in energy aware AODV protocol to improve the communication Range and hence reducing interference. In our proposed algorithm, the nodes are selected on the basis of their energy status, which help in discovering alternate paths. In proposed algorithm, neighboring nodes (backbone nodes) of active route having energy above than some threshold value are selected for route establishment between source and destination. Some modifications have been done to improve the performance of AODV. It controls the transmission power consumption of nodes by listening to only their messages. This is done by adding two fields to RREQ and RREP packets. It also increases the lifetime of a network by judging the duplicity of broadcast.

\section{Concept of Wirelessly Power Transformation}

Household devices produce relatively small magnetic fields. For this reason, chargers hold devices at the distance necessary to induce a current, which can only happen if the coils are close together. A larger, stronger field could induce current from farther away, but the process would be extremely inefficient. Since a magnetic field spreads in all directions, making a larger one would waste a lot of energy [3]. An efficient way to transfer power between coils separated by a few meters is that we could extend the distance between the coils by adding resonance to the equation. A good way to understand resonance is to think of it in terms of sound. An object's physical structure -- like the size and shape of a trumpet -- determines the frequency at which it naturally vibrates. This is its resonant frequency [14] It's easy to get objects to vibrate at their resonant frequency and difficult to get them to vibrate at other frequencies. This is why playing a trumpet can cause a nearby trumpet to begin to vibrate. Both trumpets have the same resonant frequency. Induction can take place little differently if the electromagnetic fields around the coils resonate at the same frequency. The theory uses a curved coil of wire as an inductor. A capacitance plate, which can hold a charge, attaches to each end of the coil. As electricity travels through this coil, the coil begins to resonate. Its resonant frequency is a product of the inductance of the coil and the capacitance of the plates [15]. 


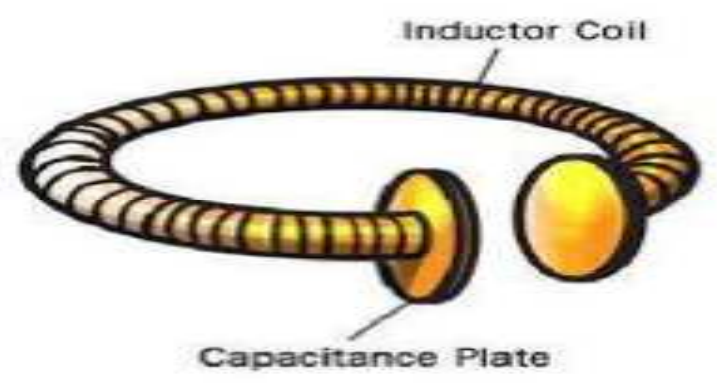

Fig. 1. Charged Coil.

Electricity, traveling along an electromagnetic wave, can tunnel from one coil to the other as long as they both have the same resonant frequency. In a short theoretical analysis they demonstrate that by sending electromagnetic waves around in a highly angular waveguide, evanescent waves are produced which carry no energy. An evanscent wave is near field standing wave exhibiting exponential decay with distance. If a proper resonant waveguide is brought near the transmitter, the evanescent waves can allow the energy to tunnel (specifically evanescent wave coupling, the electromagnetic equivalent of tunneling to the power drawing waveguide, where they can be rectified into DC power. Since the electromagnetic waves would tunnel, they would not propagate through the air to be absorbed or dissipated, and would not disrupt electronic devices. As long as both coils are out of range of one another, nothing will happen, since the fields around the coils aren't strong enough to affect much around them. Similarly, if the two coils resonate at different frequencies, nothing will happen. But if two resonating coils with the same frequency get within a few meters of each other, streams of energy move from the transmitting coil to the receiving coil. According to the theory, one coil can even send electricity to several receiving coils, as long as they all resonate at the same frequency. The researchers have named this non-radiative energy transfer since it involves stationary fields around the coils rather than fields that spread in all directions.

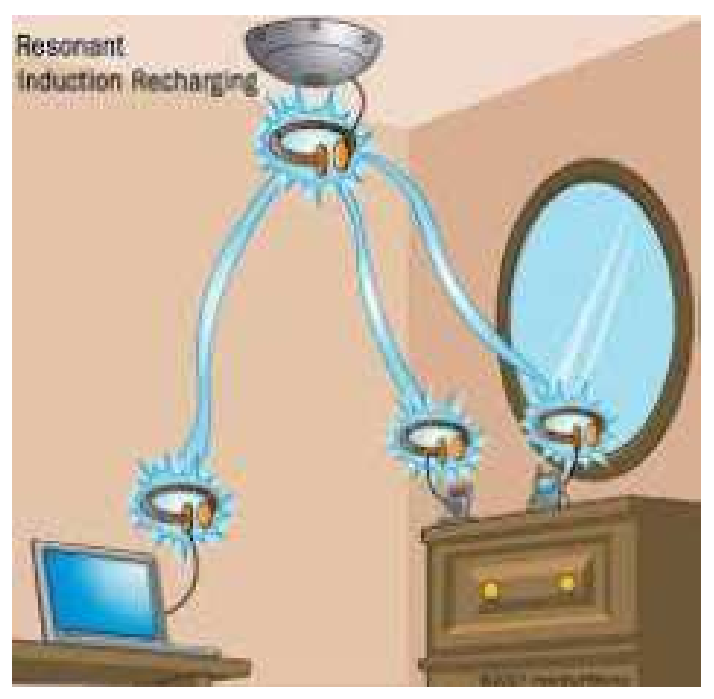

Fig. 2. Flow of charge.
According to the theory, one coil can recharge any device that is in range, as long as the coils have the same resonant frequency. "Resonant inductive coupling" has key implications in solving the two main problems associated with nonresonant inductive coupling and electromagnetic radiation, one of which is caused by the other; distance and efficiency. Electromagnetic induction works on the principle of a primary coil generating a predominantly magnetic field and a secondary coil being within that field so a current is induced within its coils. This causes the relatively short range due to the amount of power required to produce an electromagnetic field. Over greater distances the non-resonant induction method is inefficient and wastes much of the transmitted energy just to increase range. This is where the resonance comes in and helps efficiency dramatically by "tunneling" the magnetic field to a receiver coil that resonates at the same frequency. Unlike the multiple-layer secondary of a non-resonant transformer, such receiving coils are single layer solenoids with closely spaced capacitor plates on each end, which in combination allow the coil to be tuned to the transmitter frequency thereby eliminating the wide energy wasting "wave problem" and allowing the energy used to focus in on a specific frequency increasing the range.

\section{Proposed Plan}

The objective is to provide an energy efficient, more stable and long lasting path from source to destination. This has been done by modifying AODV protocol. A new routing scheme with new protocol EEAODV has been designed for mobile ad hoc network with large number of nodes. It can handle a variety of data traffic levels. This scheme is designed for network topology in which the nodes can trust each other and there are no malicious intruder nodes. The new routing scheme has been proposed to make AODV energy efficient and for all practical purposes it has been assumed that battery status is divided into 3 categories:

1) If (battery status $<20 \%$ ) It is called danger state.

2) If $(20 \%>$ battery status $<50 \%)$ it is critical state

3) If (bSattery status $>50 \%$ ) It is active state where \%age is the decay factor of battery.

There are three major operations in this scheme: RREQ (Route Request) phase, RERR (Route Errors) phase and local repair phase. As a major change to all existing protocols, power related function starts only when RREP (Route Reply) phase occurs as in the start, when network is new, all nodes are fresh with adequate energy levels and can very well select shortest path for data transmission. This in turn reduces overhead and end to end delay. So we have used this parameter in LRR (Local Repair) and RERR phase and not in RREQ phase.

\section{Proposed Algorithm}

The proposed algorithm is as under:

Step 1: The nodes which are not participating in route go to sleep mode from the start. 
Step 2: Source node $\mathrm{S}$ broadcasts an active request to the destination D. [This request is same as RREQ as used in AODV]

Step 3: Check reply phase and set active path [only nodes with status greater than critical level are selected.]

Step 4: In case of link failure, check backbone nodes (one hop) for the link failure path.

This is carried out using local repair scheme. In the route table, energy factor is added.

\section{Performance Evaluation Using Network Simulator}

The simulations have been carried out over network simulator (Fall and Varadhan, 2009) NS-2 version 2.34. The area considered is $1 \mathrm{KM} \times 1 \mathrm{KM}$ (square flat topology). The number of wireless mobile nodes was fixed to 50 nodes. The random waypoint model (Bettstetter and Hartenstein, 2002) is used to model mobility. The random scenarios have been generated with speed varying from 1 meter/second to 10 meter/second and pause time varying from 0 seconds to 500 seconds. Traffic sources are chosen as TCP-IP with a packetsize of 512 bytes. All traffic sessions are established at random times near the beginning of the simulation run and they remain active until the end of the simulation period. Simulations are run for 1000 seconds. NS-2 inbuilt $714 \mathrm{MHz}$ lucent wave LAN Direct Sequence Spread Spectrum (DSSS) model with various simulation parameters has been used for generating energy patterns.

\subsection{Simulation Results on the Basis of Packet Delivery Ratio}

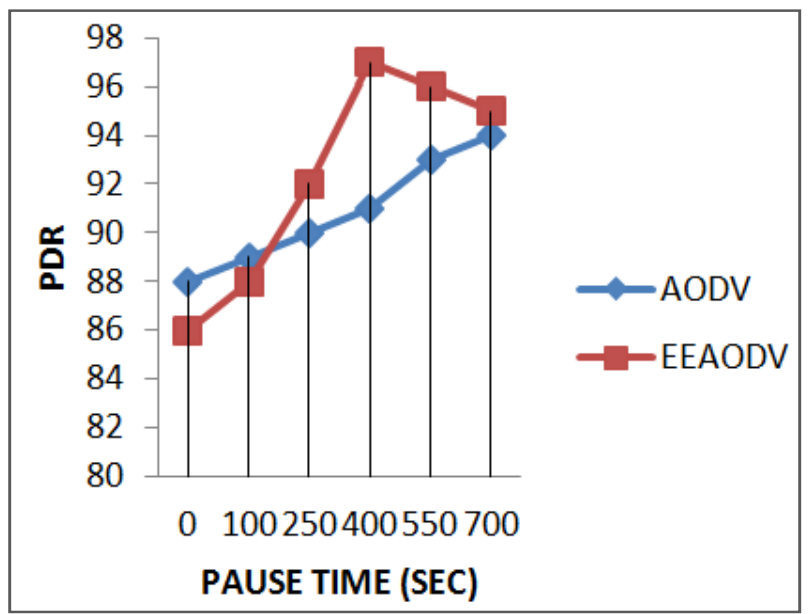

Figure 3. Pattern analysis with UDP connections and varying pause time.

Figure 3 depicts the packet delivery ratio using pause time as a parameter for AODV and EEAODV protocol. The results are on the basis of 50 mobile nodes having UDP connections. Pause time has been varied from 0 seconds to 500 seconds. The results show that, when the pause time ranges between 0 to 100, AODV outperforms EEAODV but when the pause time is further increased from 100 seconds to
500 seconds, the EEAODV starts outperforming than AODV in terms of packet delivery. On an average, EEAODV outperforms AODV protocol.

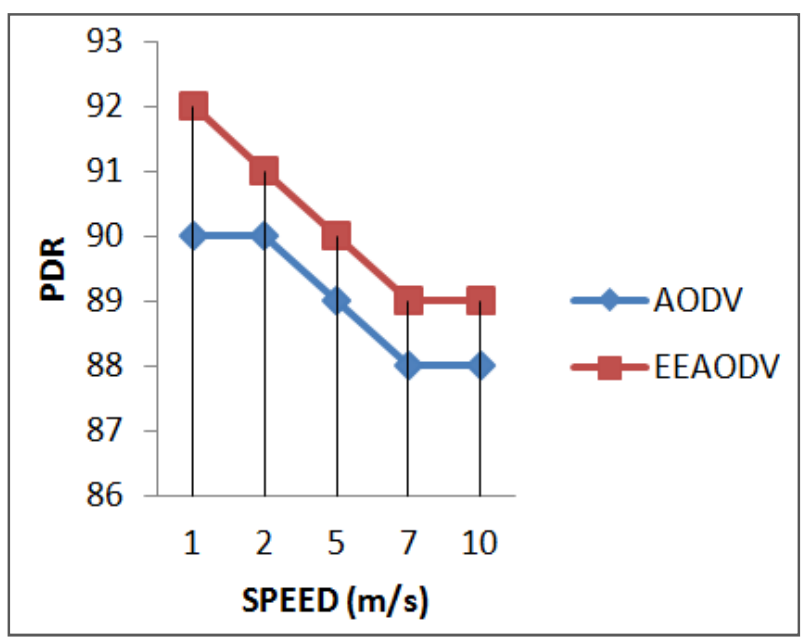

Figure 4. Pattern analysis with UDP connections and varying speed.

The packet delivery ratio using speed as a parameter for AODV and EEAODV protocol has been illustrated in Figure 4. The results are on the basis of 50 mobile nodes having UDP connections. Speed has been varied from 1 meter/second to 10 meter/second. The results show that, the EEAODV outperforms the AODV protocol irrespective of speed.

The packet delivery ratio using pause time as a parameter for AODV and EEAODV protocol is depicted in Figure 5. The results are on the basis of 50 mobile nodes having TCP connections. Pause time has been varied from 0 seconds to 500 seconds. The results show that, the EEAODV outperforms the AODV protocol irrespective of pause time.

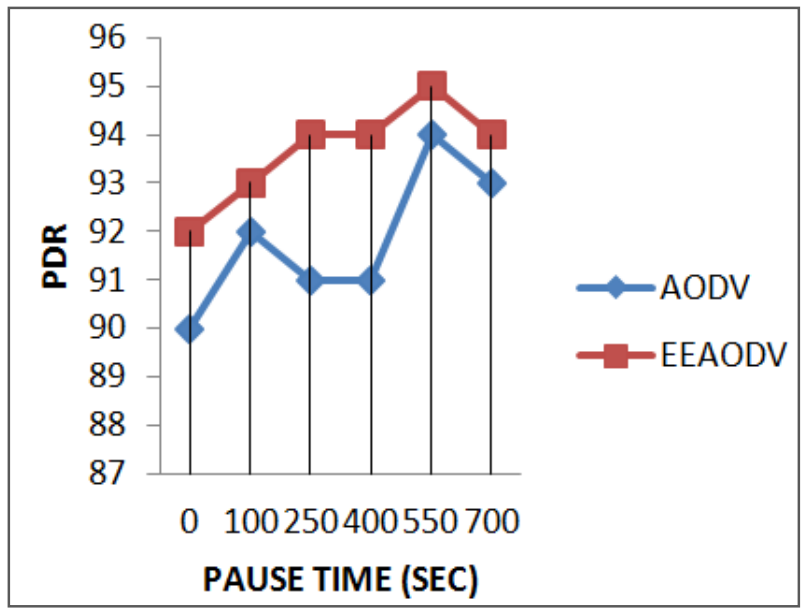

Figure 5. Pattern analysis with TCP connections and varying pause time.

Figure 6 depicts the packet delivery ratio using speed as a parameter for AODV and EEAODV protocol. The results are on the basis of 50 mobile nodes having TCP connections. Speed has been varied from 1 meter/second to 10 meter/second. The results show that, when the speed lies 
between 1 meter/second to 2 meter/seconds, EEAODV outperforms AODV but when the speed is further increased from 2 meter/second to 10 meter/second, the AODV protocol starts outperforming than EEAODV. On an average, the AODV protocol outperforms than EEAODV.

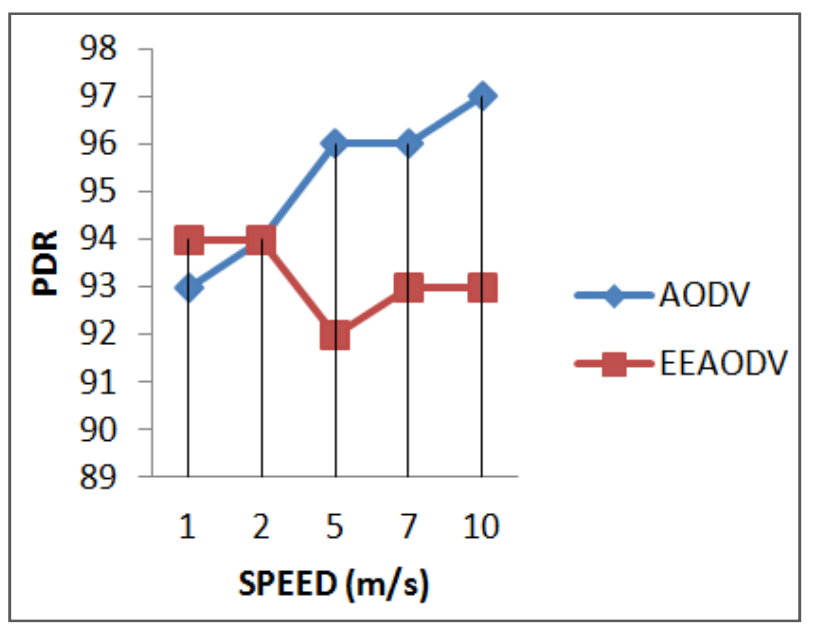

Figure 6. Pattern analysis with TCP connections and varying speed.

\subsection{Simulation Results on the Basis of Exhausted Nodes}

Exhausted nodes are the number of nodes that die out at the end of each simulation run, due to the consumption of whole energy supplied to them at the start of the simulation. The figure 7 illustrates the behavior of AODV and EEAODV for varying number of sources with respect to exhausted nodes. It can be observed that on an average, more number of nodes died till the end of simulation in case of EEAODV while less number of deaths was reported in the case of AODV.

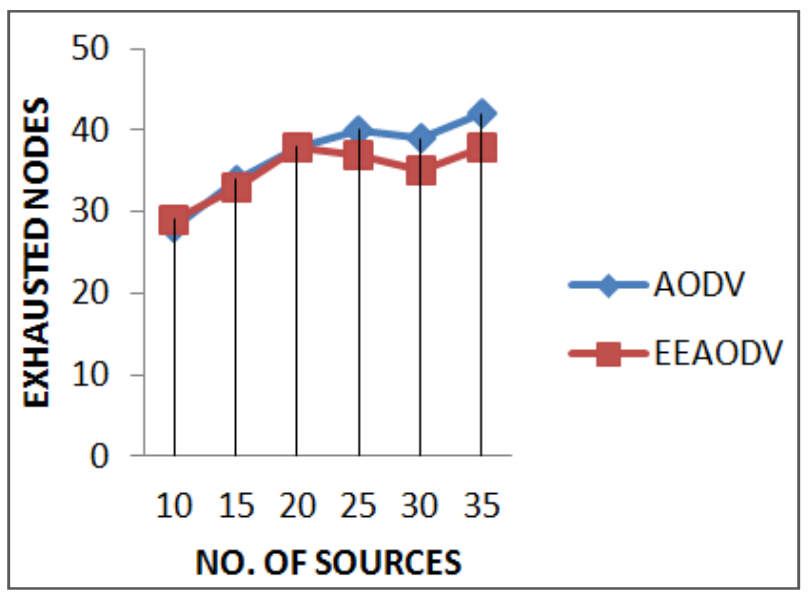

Figure 7. Pattern analysis on the basis of exhausted nodes.

\section{Conclusion and Future Scope}

Many researchers have worked in the field of power aware routing over mobile ad hoc network using efficient battery management, transmission power management and system power management. Their research work has been considered and an effort has been made to modify the existing AODV protocol by introducing power efficiency. In proposed algorithm, the route selection depends upon the battery strength of neighboring nodes which can be in danger state, critical state or active state. The EEAODV makes optimum utilization of battery strength during route selection. The results have been derived by carrying out experiments over network simulator NS-2. The performance evaluation of EEAODV and existing AODV has been done on the basis of packet delivery ratio and exhausted nodes. The proposed scheme in EEAODV works on a reactive approach and utilizes alternate paths by satisfying a set of energy based criteria. This scheme can be incorporated into any ad hoc on demand routing protocol to reduce frequent route discoveries. Alternate routes are utilized only when data cannot be delivered through the primary route. Simulation results indicate that the proposed scheme provides robustness to mobility and enhances protocol performance.

Average increase in packet delivery occurs for different network scenarios. The scheme performs better in denser medium as more nodes are available for better route selection. In this scheme, power related function starts only when route reply phase starts. This is a major change to all existing protocols. Efforts are still going on to make this scheme to work better in sparse medium and to get better results for EEAODV when TCP connections are there and nodes are moving at high speed. The work is also being carried out to get better results for EEAODV when exhausted nodes are used as parameter for performance evaluation.

\section{References}

[1] Manish Bhardwaj. Selection of Efficient Relay for EnergyEfficient Cooperative Ad Hoc Networks. American Journal of Networks and Communications. Special Issue: Ad Hoc Networks. Vol. 4, No. 3-1, 2015, pp. 5-11. doi: 10.11648/j.ajnc.s. 2015040301.12

[2] Megha Sharma, Shivani Rohilla, Manish Bhardwaj. Efficient Routing with Reduced Routing Overhead and Retransmission of Manet. American Journal of Networks and Communications. Special Issue: Ad Hoc Networks. Vol. 4, No. 3-1, 2015, pp. 22-26. doi: 10.11648/j.ajnc.s. 2015040301.15

[3] Manish Bhardwaj, Enhance life Time of Mobile Ad hoc Network using WiTriCity and Backpressure Technique, 18770509 (C) 2015 The Authors. Published by Elsevier B.V. This is an open access article under the CC BY-NC-ND license, doi: 10.1016/j.procs.2015.07.447.

[4] A. Kurs, A. Karalis, R. Moffatt, J. D. Joannopoulos, P. Fisher, M. Soljacic. 2008. Wireless Power Transfer via Strongly Coupled Magnetic Resonances. Science. http://www.sciencemag.org/cgi/rapid.df/1143254?ijkey=94ff. Ay4jRMqU\&keytype $=$ ref\&siteid=s ci (accessed December 5, 2011).

[5] A. Kuris et al., "Wireless Power Transfer via Strongly Coupled Magnetic Resonances," Journal Science, vol. 317, pp 83-85, July 2007. 
[6] Adamou M. and Sarkar S. (2002). A Framework for Optimal Battery Management for Wireless Nodes. Proceedings of IEEE INFOCOMP, 1783-1792.

[7] Bettstetter C. and Hartenstein H. Stochastic. (2002). Properties of the Random Waypoint Mobility Model: Epoch Length, Direction Distribution and Cell Change Rate. Proceedings of the 5th ACM International Workshop on Modelling Analysis and Simulation of Wireless and Mobile Systems, 7-14.

[8] C. E. Perkins and E. M. Royer. (1999). Ad-Hoc On Demand Distance Vector Routing. Proceedings of the 2nd IEEE Workshop on Mobile Computing Systems and Applications (WMCSA), 90-100.

[9] Chang J. H. and Tassiulas L. (2000). Energy Conserving Routing in Wireless Ad hoc Networks. Proceedings of IEEE INFOCOM, 22-31.

[10] Chen Jie, Chen Jiapin, Li Zhenbo. (2007). Energy Efficient AODV for low mobility Ad hoc Network. Proceedings of International Conference on Wireless Communications, Networking and Mobile Computing, WiCom 2007, 1512-1515.

[11] Chiasserini C. F., Chlamtac I., Monti P. and Nucci A. (2002). Energy Efficient Design of Wireless Ad hoc Networks. Proceedings of Networking, 376- 386.

[12] Jayashree S., Manoj B.S. and Siva Ram Murthy C. (2003). Energy Management in Ad hoc Wireless Networks: A Survey of Issues and Solutions. Technical Report, Department of Computer Science and Engineering, Indian Institute of Technology, Madras, India.

[13] Jin-Man Kim, Jong-Wook Jang. (2006). AODV based Energy Efficient Routing Protocol for Maximum Lifetime in MANET. Advanced International Conference on Telecommunications and International Conference on Internet and Web Applications and Services (AICT-ICIW'06), 77.

[14] Johnson D.B. and Maltz D.B. (1996). Dynamic Source Routing in Ad hoc Wireless Networks. Kluwer Academic Publishers, 353, 153-181.

[15] Kawadia V. and Kumar P. R. (2003). Power Control and Clustering in Ad hoc Networks. Proceedings of IEEE INFOCOM'03, 459-469.
[16] Kuo-Qin Yan, Shu-Ching Wang, Mao-Lun Chiang and Lin-Yu Tseng (2009). A Fuzzy-based Power-aware Management for Mobile Ad hoc Networks, ACM Digital Library, Journal Computer Standards \& Interfaces, Volume 31, Issue 1, January, 2009.

[17] Nie Nie and Cristina Comaniciu (2006). Energy efficient AODV routing in CDMA Ad hoc networks using Beamforimg. EURASIP Journal on Wireless Communications and Networking , 2006(2).

[18] Kevin Fall and Kannan Varadhan (2009). The $n s$ Manual, The VINT Project, available at http://www.isi.edu/nsnam/ns/ns documentation.html

[19] Radhika D. Joshi and Priti P. Rege. (2007). Energy Aware Routing in Ad hoc network. Proceedings of the Sixth International Conference on Circuits, Systems, Electronics, Control and Signal Processing (WSEAS), 469-475.

[20] Royer E. M. and Toh C. K. (1999). A Review of Current Routing Protocols for Ad hoc Mobile Wireless Networks. IEEE Personal Communications, 6(2), 46-55.

[21] Singh S. and Raghavendra C. S. (1998). PAMAS: Power Aware Multi-Access protocol with Signalling for Ad-Hoc Networks. ACM SIGCOMM, Computer Communication Review, 5-26.

[22] Taneja, S., A. Kush and A. Makkar. (2010). Mobility Model for Power Based AODV. International Journal of Information Sciences \& Application (IJISA), 2(2), 251-257.

[23] Toh C. K. (2001). Maximum Battery Life Routing to Support Ubiquitous Mobile Computing in Wireless Ad hoc Networks. IEEE Communications Magazine, 39(6), 138-147.

[24] Woo M., S. Singh. and Ragh 'avendra C. S. (1998). PowerAware Routing in Mobile Ad hoc Networks. Proceedings of ACM/IEEE International Conference on Mobile Computing and Networking, 181-190.

[25] Zheng R. and Kravets R. (2003). On Demand Power Management for Ad hoc Networks. Proceedings of IEEE INFOCOMP 2003, 1, 481-491. 